Теорія Ймовір. та Матем. Статист. Вип. 81,2010
Theor. Probability and Math. Statist.

No. 81, 2010, Pages 187-195 S 0094-9000(2011)00819-8

Article electronically published on January 24, 2011

\title{
SINGULARITY OF THE SECOND OSTROGRADSKIĬ RANDOM SERIES
}

UDC 519.21

\author{
G. M. TORBIN AND I. M. PRATSYOVYTA
}

\begin{abstract}
We study properties of the distribution of the second Ostrogradskil series, for which the differences of terms are independent identically distributed random variables. We completely describe the Lebesgue structure of this distribution. In particular, we prove that it cannot be absolutely continuous. We also develop ergodic theory for the second Ostrogradskil expansion. One of the results is that, for almost all (in the sense of Lebesgue measure) real numbers of the unit interval, an arbitrary symbol of an alphabet occurs finitely often in the corresponding Ostrogradskiu difference expansion. We also study properties of the dynamical system generated by the one-sided shift transformations $T$ of the Ostrogradskil difference representation. It is shown that there is no probability measure that is invariant and ergodic with respect to $T$ and absolutely continuous with respect to Lebesgue measure.
\end{abstract}

\section{INTRODUCTION}

In the early 1860s, M. V. Ostrogradskil considered two algorithms for expansions of positive real numbers into sign-alternating series:

$$
\begin{gathered}
\sum_{k} \frac{(-1)^{k-1}}{q_{1} q_{2} \cdots q_{k}}, \quad \text { where } q_{k} \in \mathbb{N}, q_{k+1}>q_{k}, \\
\sum_{k} \frac{(-1)^{k-1}}{q_{k}}, \quad \text { where } q_{k} \in \mathbb{N}, q_{k+1} \geq q_{k}\left(q_{k}+1\right)
\end{gathered}
$$

(called the first and second Ostrogradskiur series, respectively). His brief notes on the matter were discovered in The Manuscript Depository of the National Academy of Sciences of Ukraine and explained by E. Ya. Remez in 1951 (see [13, where it is shown that Ostrogradskin's algorithms give better approximations than continued fractions in certain cases).

In a footnote in the Khinchin book [14, B. V. Gnedenko wrote “... Unfortunately, no detailed study of these algorithms, even for computational purposes, has as yet been made." It is worth mentioning that nowadays there is a number of researches (see [1, 2, 7, 8, 9, 11, 12 and further references therein) devoted to expansions of real numbers into the sign-alternating first Ostrogradskil series (also known as Pierce series) and to probability distributions related to these series.

2010 Mathematics Subject Classification. Primary 11K55, 37B10, 60G30.

Key words and phrases. The second Ostrogradskiu series, singular probability measures, symbolic dynamics.

The research of the first author is supported by the projects DFG 436 UKR 113/80 and DFG 436 113/97 and the Alexander von Humboldt Foundation.

The research of the second author is supported by the projects DFG 436 UKR 113/80 and DFG 436 $113 / 97$. 
The second Ostrogradskiŭ expansions are much less studied ([10, 11, 13]). In particular, it is known that every real number $x \in(0,1)$ can be represented as a finite or infinite expression of the form (11). If a number $x$ is irrational, then this representation is unique and the series in (2) is infinite, while if $x$ is rational, then there are two different representations of the form (2) and both of them are finite. The Ostrogradskil series converges quickly and this allows one to approximate irrational numbers effectively by partial sums of their Ostrogradskil series. Note that the partial sums are rational numbers.

The following representation of a number $x$ :

$$
x=\sum_{k} \frac{(-1)^{k-1}}{q_{k}(x)}, \quad \text { where } q_{k}(x) \in \mathbb{N}, q_{k+1}(x) \geq q_{k}(x)\left(q_{k}(x)+1\right),
$$

is called the $O^{2}$-representation of $x$ and is denoted by $O^{2}\left(q_{1}(x), \ldots, q_{n}(x), \ldots\right)$.

If the initial $k$ terms are fixed, the $(k+1)$ th term of the $O^{2}$-representation cannot equal $1,2,3,4,5, \ldots, q_{k}\left(q_{k}+1\right)-1$, which shows that the terms of an $O^{2}$-representation have "unequal rights". Let

$$
d_{1}=q_{1} \quad \text { and } \quad d_{k+1}=q_{k+1}-q_{k}\left(q_{k}+1\right)+1 \quad \text { for all } k \in \mathbb{N} .
$$

Then the latter series can be rewritten as follows:

$$
x=\sum_{k} \frac{(-1)^{k+1}}{q_{k-1}(x)\left(q_{k-1}(x)+1\right)-1+d_{k}(x)}=: \bar{O}^{2}\left(d_{1}(x), d_{2}(x), \ldots, d_{k}(x), \ldots\right) .
$$

The expression on the right hand side of (4) is called the $\bar{O}^{2}$-expansion (other names are the second Ostrogradskil difference expansion and the second Ostrogradskiı expansion with independent increments), and the number $d_{k}=d_{k}(x)$ is called the $k$ th $\bar{O}^{2}$-symbol of the number $x$. In a $\bar{O}^{2}$-representation, any symbol, independently of the values of the preceding one, may attain every natural value.

The main goals in this paper are:

1) To develop an ergodic theory of $\bar{O}^{2}$-expansions of real numbers expressed in terms of asymptotic frequencies $\nu_{i}\left(x, \bar{O}^{2}\right)$ of $\bar{O}^{2}$-symbols $(i \in \mathbb{N})$, where

$$
\nu_{i}\left(x, \bar{O}^{2}\right)=\lim _{n \rightarrow \infty} \frac{N_{i}(x, n)}{n}
$$

and where $N_{i}(x, n)$ is the number of $i$ 's in the $\bar{O}^{2}$-representation of a number $x$ up to the $n$th position; in particular, to find normal properties of real numbers (in other words, those properties that hold for almost all real numbers with respect to Lebesgue measure).

2) To investigate properties of the dynamical system generated by the following onesided shift transformation $T$ in the $\bar{O}^{2}$-representation:

$$
\begin{gathered}
T(x)=T\left(\bar{O}^{2}\left(d_{1}(x), d_{2}(x), \ldots, d_{n}(x), \ldots\right)\right)=\bar{O}^{2}\left(d_{2}(x), d_{3}(x), \ldots, d_{n}(x), \ldots\right) \\
\quad \text { for all } x=\bar{O}^{2}\left(d_{1}(x), d_{2}(x), \ldots, d_{n}(x), \ldots\right) \in[0,1]
\end{gathered}
$$

3) To study properties of the random variable

$$
\eta=\bar{O}^{2}\left(\eta_{1}, \eta_{2}, \ldots, \eta_{k}, \ldots\right),
$$

whose $\bar{O}^{2}$-symbols $\eta_{k}$ are independent identically distributed random variables assuming values $1,2, \ldots, m, \ldots$ with probabilities $p_{1}, p_{2}, \ldots, p_{m}, \ldots$, respectively, where the sequence $\left\{p_{m}\right\}$ is such that $p_{m} \geq 0$ and $\sum_{m=1}^{\infty} p_{m}=1$. 


\section{Geometry of an $O^{2}$-representation and of a $\bar{O}^{2}$-representation OF REAL NUMBERS}

Let $\left(c_{1}, c_{2}, \ldots, c_{n}\right)$ be a given family of positive integers. The set of numbers $x \in(0,1]$ of the form

$$
\Delta_{c_{1} c_{2} \ldots c_{n}}^{O^{2}}=\left\{x: x=O^{2}\left(q_{1}, q_{2}, \ldots, q_{n}, \ldots\right), q_{i}=c_{i}, i=1, \ldots, n, q_{n+j} \in \mathbb{N}\right\}
$$

is called the cylinder of rank $n$ with base $c_{1} c_{2} \ldots c_{n}$.

Below we list some properties of cylinder sets.

1. $\Delta_{c_{1} \ldots c_{n}}^{O^{2}}=\bigcup_{i=c_{n}\left(c_{n}+1\right)}^{\infty} \Delta_{c_{1} \ldots c_{n} i}^{O^{2}}$.

2 .

$$
\begin{gathered}
\inf \Delta_{c_{1} \ldots 2 m-1}^{O^{2}}=\sum_{k=1}^{2 m-1} \frac{(-1)^{k-1}}{c_{k}}-\frac{1}{c_{2 m-1}\left(c_{2 m-1}+1\right)} \\
=O^{2}\left(c_{1}, \ldots, c_{2 m-1}, c_{2 m-1}\left(c_{2 m-1}+1\right)\right) \\
=O^{2}\left(c_{1}, \ldots, c_{2 m-2}, c_{2 m-1}+1\right) \in \Delta_{c_{1} \ldots c_{2 m-1}}^{O^{2}} ; \\
\sup \Delta_{c_{1} \ldots 2 m-1}^{O^{2}}=\sum_{k=1}^{2 m-1} \frac{(-1)^{k-1}}{c_{k}}=O^{2}\left(c_{1}, \ldots, c_{2 m-1}\right) \in \Delta_{c_{1} \ldots c_{2 m-1}}^{O^{2}} ; \\
\inf \Delta_{c_{1} \ldots 2 m}^{O^{2}}=\sum_{k=1}^{2 m} \frac{(-1)^{k-1}}{c_{k}}=O^{2}\left(c_{1}, \ldots, c_{2 m}\right) \in \Delta_{c_{1} \ldots c_{2 m}}^{O^{2}} ; \\
\sup \Delta_{c_{1} \ldots 2 m}^{O^{2}}=\sum_{k=1}^{2 m} \frac{(-1)^{k-1}}{c_{k}}+\frac{1}{c_{2 m}\left(c_{2 m}+1\right)}=O^{2}\left(c_{1}, \ldots, c_{2 m}, c_{2 m}\left(c_{2 m}+1\right)\right) \\
=O^{2}\left(c_{1}, \ldots, c_{2 m-1}, c_{2 m}+1\right) \in \Delta_{c_{1} \ldots c_{2 m}}^{O^{2}} .
\end{gathered}
$$

3.

$$
\begin{gathered}
\sup \Delta_{c_{1} \ldots c_{2 m-1} i}^{O^{2}}=\inf \Delta_{c_{1} \ldots c_{2 m-1}(i+1)}^{O^{2}} ; \\
\inf \Delta_{c_{1} \ldots c_{2 m} i}^{O^{2}}=\sup \Delta_{c_{1} \ldots c_{2 m}(i+1)}^{O^{2}} .
\end{gathered}
$$

4. The length of a cylinder interval of rank $n$ satisfies

$$
\left|\Delta_{c_{1} \ldots c_{n}}^{O^{2}}\right|=\frac{1}{c_{n}\left(c_{n}+1\right)} \rightarrow 0, \quad n \rightarrow \infty .
$$

5. The length of a cylinder depends on the last symbol of the basis only, that is,

$$
\left|\Delta_{c_{1} \ldots c_{n} i}^{O^{2}}\right|=\frac{1}{i(i+1)}=\left|\Delta_{s_{1} \ldots s_{m} i}^{O^{2}}\right| \text {. }
$$

6.

$$
\frac{\left|\Delta_{c_{1} \ldots c_{n} c_{n+1}}^{O^{2}}\right|}{\left|\Delta_{c_{1} \ldots c_{n}}^{O^{2}}\right|}=\frac{c_{n}\left(c_{n}+1\right)}{c_{n+1}\left(c_{n+1}+1\right)} \leq \frac{1}{2^{2^{n-1}}} \quad \text { for all } n \in \mathbb{N} .
$$

Any of the cylinders of an $O^{2}$-representation can be rewritten in terms of a $\bar{O}^{2}$ representation as follows:

$$
\Delta_{c_{1} \ldots c_{n}}^{O^{2}} \equiv \bar{\Delta}_{a_{1} \ldots a_{n}}^{O^{2}}
$$

where $a_{1}=c_{1}, a_{k}=c_{k}+1-c_{k-1}\left(c_{k-1}+1\right), 1<k<n$.

Properties of the cylinders corresponding to an $O^{2}$-representation are analogous to those of cylinders corresponding to a $\bar{O}^{2}$-representation. We mention only one of those properties:

$$
\left|\Delta_{a_{1} \ldots a_{n} a_{n+1}}^{\bar{O}^{2}}\right| \leq \frac{1}{2^{2^{n-1}}}\left|\Delta_{a_{1} \ldots a_{n}}^{\bar{O}^{2}}\right| \quad \text { for all } n \in \mathbb{N}
$$




\section{Normal Properties of numbers AND their $\bar{O}^{2}$-REPRESEntations}

A property of real numbers is called normal if it holds for almost all (in the sense of Lebesgue measure) real numbers. Examples of normal properties are given by the properties "to be irrational" or "to be transcendental," which do not depend on the chosen number system (in other words, on the way the real numbers are represented). If a representation of real numbers is fixed, their normal properties can be conveniently expressed in terms of symbols (digits) in their representation. For example, the following are normal properties if one uses the decimal expansions of numbers: "to contain infinitely many zeros in the decimal expansion", "to be aperiodic", "to contain every digit with frequency $10^{-1}$," and so on. If one uses the representation in the form of continued fractions, then the following are also examples of normal properties: "to have infinitely many elements in the representation", "to contain a symbol $i$ with asymptotic frequency $\frac{1}{\ln 2} \ln \frac{(i+1)^{2}}{i(i+2)}$," and so on.

The main goal in this section is to find some normal properties of real numbers expressed in terms of the sequence $\left\{d_{k}(x)\right\}$ for the second Ostrogradskiu difference expansion.

Theorem 3.1. Almost all (in the sense of Lebesgue measure) numbers of the interval $(0,1]$ contain every $\bar{O}^{2}$-symbol finitely many times in their $\bar{O}^{2}$-representation.

Proof. Let $N_{i}(x)$ be the number of occurrences of a symbol $i$ in the $\bar{O}^{2}$-representation of a number $x$. We prove that the Lebesgue measure of the set $A_{i}=\left\{x: N_{i}(x)=\infty\right\}$ is equal to zero for all $i \in N$.

Consider the set

$$
\bar{\Delta}_{i}^{k}=\left\{x: x=\bar{O}^{2}\left(d_{1}, \ldots, d_{k-1}, i, d_{k+1}, \ldots\right), d_{j} \in \mathbb{N} \text { for all } j \neq k\right\}
$$

of numbers of the interval $(0,1]$ for which the symbol $i$ occurs at the position $k$ of the $\bar{O}^{2}$-representation, that is, $d_{k}(x)=i$.

Lemma 3.1. For all positive integers $i$ and $k$, we have

$$
\lambda\left(\bar{\Delta}_{i}^{1}\right)=\frac{1}{i(i+1)} \leq \frac{1}{2}, \quad \lambda\left(\bar{\Delta}_{i}^{k}\right) \leq \frac{1}{2^{2^{k-2}}} \quad \text { for } k>1 .
$$

Proof. Since

$$
\bar{\Delta}_{i}^{1}=\Delta_{i}^{\bar{O}^{2}}=\left[\frac{1}{i+1}, \frac{1}{i}\right]
$$

we obtain $\lambda\left(\bar{\Delta}_{i}^{1}\right)=(i(i+1))^{-1} \leq \frac{1}{2}$.

Properties 1 and 6 of cylinder sets listed above and the definition of the set $\bar{\Delta}_{i}^{k}$ imply that

$$
\bar{\Delta}_{i}^{k}=\bigcup_{a_{1}=1}^{\infty} \ldots \bigcup_{a_{k-1}=1}^{\infty} \Delta_{a_{1} \ldots a_{k-1} i}^{\bar{O}^{2}} \quad \text { and } \quad \frac{\left|\Delta_{a_{1} \ldots a_{k-1} i}^{\bar{O}^{2}}\right|}{\left|\Delta_{a_{1} \ldots a_{k-1}}^{\bar{O}^{2}}\right|} \leq \frac{1}{2^{2^{k-2}}}
$$

Thus

$$
\begin{aligned}
\lambda\left(\bar{\Delta}_{i}^{k}\right) & =\sum_{a_{1}=1}^{\infty} \ldots \sum_{a_{k-1}=1}^{\infty}\left|\Delta_{a_{1} \ldots a_{k-1} i}^{\bar{O}^{2}}\right| \leq \frac{1}{2^{2^{k-2}}}\left(\sum_{a_{1}=1}^{\infty} \cdots \sum_{a_{k-1}=1}^{\infty}\left|\Delta_{a_{1} \ldots a_{k-1}}^{\bar{O}^{2}}\right|\right) \\
& =\frac{1}{2^{2^{k-2}}} .
\end{aligned}
$$


It is obvious that $A_{i}$ is the upper limit of the sequence of sets $\left\{\bar{\Delta}_{i}^{k}\right\}$, that is,

$$
A_{i}=\limsup _{k \rightarrow \infty} \bar{\Delta}_{i}^{k}=\bigcap_{m=1}^{\infty}\left(\bigcup_{k=m}^{\infty} \bar{\Delta}_{i}^{k}\right) \text {. }
$$

Since

$$
\sum_{k=1}^{\infty} \lambda\left(\bar{\Delta}_{i}^{k}\right) \leq \sum_{k=1}^{\infty} \frac{1}{2^{2^{k-2}}}<+\infty
$$

the Borel-Cantelli lemma implies that

$$
\lambda\left(A_{i}\right)=0 \quad \text { for all } i \in \mathbb{N} .
$$

Hence

$$
\lambda\left(\bar{A}_{i}\right)=1 \quad \text { for all } i \in \mathbb{N}
$$

Let

$$
\bar{A}=\bigcap_{i=1}^{\infty} \bar{A}_{i}
$$

It is obvious that $\lambda(\bar{A})=1$. The theorem is proved.

Let $N_{i}(x, k)$ be the number of occurrences of the symbol $i$ up to the position $k$ in the $\bar{O}^{2}$-representation of a number $x$. If the $\operatorname{limit}_{k \rightarrow \infty} N_{i}(x, k) / k$ exists, it is called the asymptotic frequency of the digit (symbol) $i$ in the $\bar{O}^{2}$-representation of a number $x$. The asymptotic frequency is denoted by $\nu_{i}\left(x, \bar{O}^{2}\right)$ or simply $\nu_{i}(x)$, if it is clear from the context what it stays for.

Corollary 3.1. For $\lambda$-almost all $x$ and for all $i \in \mathbb{N}$,

$$
\nu_{i}(x)=0 \text {. }
$$

Corollary 3.2. For an arbitrary random vector $\vec{p}=\left(p_{1}, p_{2}, \ldots, p_{k}, \ldots\right)$, the set

$$
I_{\vec{p}}=\left\{x: x=\bar{O}^{2}\left(d_{1}(x), \ldots, d_{k}(x), \ldots\right), \nu_{i}(x)=p_{i} \text { for all } i \in \mathbb{N}\right\}
$$

has zero Lebesgue measure.

Corollary 3.3. Let $V=\{1,2, \ldots, n\}$. Then, for an arbitrary set $V \subset N$, the set $C\left[\bar{O}^{2}, V\right]=\left\{x: x=\bar{O}^{2}\left(d_{1}, d_{2}, \ldots, d_{n}, \ldots\right), d_{j}(x) \in V\right\}$ has zero Lebesgue measure.

Let $B\left(\bar{O}^{2}\right)$ be the set of all real numbers with bounded $\bar{O}^{2}$-symbols. In other words, $x \in B\left(\bar{O}^{2}\right)$ if and only if there exists a number $K(x)$ such that $d_{k}(x) \leq K(x)$ for all $k \in \mathbb{N}$.

Corollary 3.4. The set $B\left(\bar{O}^{2}\right)$ of all numbers with bounded $\bar{O}^{2}$-symbols has zero Lebesgue measure.

\section{Singularity of the Distributions of RANDOM VARIABles REPRESEnted By THE SECOND OSTROGRADSKII SERIES WITH INDEPENDENT INCREMENTS AND PROPERTIES OF THE CORRESPONDING SYMBOLIC DYNAMICS}

Consider the following random variable:

$$
\eta=\bar{O}^{2}\left(\eta_{1}, \eta_{2}, \ldots, \eta_{k}, \ldots\right)
$$

whose $\bar{O}^{2}$-symbols $\eta_{k}$ are independent identically distributed random variables assuming values $1,2, \ldots, m, \ldots$ with probabilities $p_{1 k}, p_{2 k}, \ldots, p_{m k}, \ldots$, respectively. This means that

$$
\mathrm{P}\left\{\eta_{k}=m\right\}=p_{m k}, \quad p_{m k} \geq 0, \quad \sum_{m=1}^{\infty} p_{m k}=1 \quad \text { for all } k \in \mathbb{N} \text {. }
$$


It is obvious that the distribution of the random variable $\eta$ is completely determined by the matrix $P=\left\|p_{m k}\right\|$. A criterion for the distribution of the random variable $\eta$ to be purely discrete is given in [11. Sufficient conditions for this distribution to be singular in Cantor's sense are also presented in [11. The main goal in this section is to discover the Lebesgue structure of the distribution of the random variable $\eta$ in the case where the members of the sequence $\left\{\eta_{k}\right\}$ are independent and identically distributed.

Consider the dynamical system generated by the following one-sided shift transformation $T$ in the $\bar{O}^{2}$-representation:

$$
\begin{gathered}
T(x)=T\left(\bar{O}^{2}\left(d_{1}(x), d_{2}(x), \ldots, d_{n}(x), \ldots\right)\right)=\bar{O}^{2}\left(d_{2}(x), d_{3}(x), \ldots, d_{n}(x), \ldots\right) \\
\quad \text { for all } x=\bar{O}^{2}\left(d_{1}(x), d_{2}(x), \ldots, d_{n}(x), \ldots\right) \in[0,1] .
\end{gathered}
$$

Recall that a set $A$ is called invariant or fixed with respect to a transformation $T$ if $A=T^{-1} A$. A measure $\mu$ is called ergodic with respect to the transformation $T$ if an arbitrary invariant measure $A \in \mathfrak{B}$ is of either zero or complete measure. A measure $\mu$ is called invariant with respect to the transformation $T$ if $\mu\left(T^{-1} E\right)=\mu(E)$ for an arbitrary set $E \in \mathfrak{B}$.

Lemma 4.1. The measure $\mu_{\eta}$ is invariant and ergodic with respect to the shift transformation $T$ defined above.

Proof. 1) Let $A$ be a set fixed with respect to the transformation $T$, that is,

$$
T^{-1} A=A, \quad A \in \mathfrak{B} \text {. }
$$

Then $T\left(T^{-1} A\right)=T(A)$, whence $A=T A$. Thus $A=T^{-1} A=T^{-1}(T A)$.

If $x=\bar{O}^{2}\left(d_{1}(x), d_{2}(x), \ldots, d_{k}(x), \ldots\right)$ and $x \in A$, then

$$
T^{-1}(T x)=\left\{x: x=\bar{O}^{2}\left(c_{1}, d_{2}(x), \ldots, d_{k}(x), \ldots\right), c_{1} \in \mathbb{N}\right\} \subset A .
$$

Hence a point $x$ belongs to the invariant set $A$ independently of the first $\bar{O}^{2}$-symbol of $x$. Similarly we prove that a point $x$ belongs to the fixed set $A$ independently of the initial $n$ of the $\bar{O}^{2}$-symbols of $x$ whatever $n \geq 1$ is. Thus the Kolmogorov 0-1 law implies that either $\mu_{\eta}(A)=0$ or $\mu_{\eta}(A)=1$. Hence $\mu_{\eta}$ is ergodic with respect to the transformation $T$.

2) Since the Borel $\sigma$-algebra $\mathcal{B}$ is generated by the system of cylinders of the $\bar{O}^{2}$ representation, that is, by the system of sets of the form $\Delta_{c_{1} c_{2} \ldots c_{n}}$, it suffices to show that the measure $\mu_{\eta}$ is invariant on these cylinders. It is obvious that

$$
\mu_{\eta}\left(\Delta_{c_{1} c_{2} \ldots c_{n}}^{\bar{O}^{2}}\right)=p_{c_{1}} \cdot p_{c_{2}} \cdots p_{c_{n}}
$$

Since

$$
T^{-1}\left(\Delta_{c_{1} c_{2} \ldots c_{n}}^{\bar{O}^{2}}\right)=\Delta_{i c_{1} c_{2} \ldots c_{n}}^{\bar{O}^{2}}, \quad i \in \mathbb{N}
$$

we derive that

$$
\begin{aligned}
\mu_{\eta}\left(T^{-1}\left(\Delta_{c_{1} c_{2} \ldots c_{n}}^{\bar{O}^{2}}\right)\right) & =\sum_{i=1}^{\infty} \mu_{\eta}\left(\Delta_{i c_{1} c_{2} \ldots c_{n}}^{\bar{O}^{2}}\right)=p_{c_{1}} \cdot p_{c_{2}} \cdots p_{c_{n}} \sum_{i=1}^{\infty} p_{i} \\
& =p_{c_{1}} \cdot p_{c_{2}} \cdots p_{c_{n}}=\mu_{\eta}\left(\Delta_{c_{1} c_{2} \ldots c_{n}}^{\bar{O}^{2}}\right)
\end{aligned}
$$

and this is what was to be proved.

Lemma 4.2. For $\mu_{\eta}$-almost all $x \in[0,1]$, we have

$$
\nu_{i}\left(x, \bar{O}^{2}\right)=p_{i} \quad \text { for all } i \in \mathbb{N} .
$$


Proof. Let $T^{j}(x)$ denote the $j$ th iteration of the above shift transformation $T$. Since the measure $\mu_{\eta}$ is ergodic and invariant with respect to $T$, the Birkhoff ergodic theorem implies that

$$
\lim _{n \rightarrow \infty} \frac{1}{n} \sum_{j=0}^{n-1} \varphi\left(T^{j}(x)\right)=\int_{0}^{1} \varphi(x) d\left(\mu_{\eta}(x)\right)
$$

for $\mu_{\eta}$-almost all $x \in[0,1]$ and for an arbitrary function $\varphi \in L^{1}\left([0,1], d \mu_{\eta}\right)$.

Fix a positive integer $i$. Let $\Delta_{i}^{\bar{O}^{2}}$ be the corresponding cylinder of the first rank of the $\bar{O}^{2}$-representation. We take for a function $\varphi$ the indicator of the set $\Delta_{i}^{\bar{O}^{2}}$, that is, $\varphi(x)=1$ for $x \in \Delta_{i}^{\bar{O}^{2}}$ and $\varphi(x)=0$ otherwise.

Then

$$
\begin{gathered}
\frac{1}{n} \sum_{j=0}^{n-1} \varphi\left(T^{j}(x)\right)=\frac{N_{i}(x, n)}{n}, \\
\int_{0}^{1} \varphi(x) d\left(\mu_{\eta}(x)\right)=\int_{\Delta_{i}{ }^{2}} d\left(\mu_{\eta}(x)\right)=p_{i} .
\end{gathered}
$$

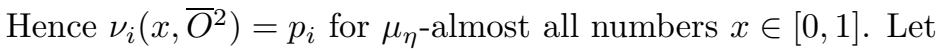

$$
M_{\left(p_{1}, p_{2}, \ldots, p_{k}, \ldots\right)}=\left\{x: x \in[0,1], \nu_{i}\left(x, \bar{O}^{2}\right)=p_{i} \text { for all } i \in \mathbb{N}\right\} .
$$

The $\mu_{\eta}$-measure of $M_{\left(p_{1}, p_{2}, \ldots, p_{k}, \ldots\right)}$ is equal to 1 , since this set is the intersection of a countable number of the sets

$$
M_{i}=\left\{x: x \in[0,1], \nu_{i}\left(x, \bar{O}^{2}\right)=p_{i}\right\}
$$

and each of them is of a complete $\mu_{\eta}$-measure.

The following theorem completely answers the question on the Lebesgue structure of the distribution of the random variable $\eta$ for the case where the members of the sequence $\left\{\eta_{k}\right\}$ are identically distributed.

Theorem 4.1. Let $\left\{\eta_{k}\right\}$ be a sequence of independent identically distributed random variables assuming values $1,2,3, \ldots$ with probabilities $p_{1}, p_{2}, p_{3}, \ldots$ and let $\sum_{i=1}^{\infty} p_{i}=1$. Then the distribution of the random variable $\eta$ of the second Ostrogradskiu representation with independent increments (that is, the random variable defined by equality (6)) is

1) either a degenerate discrete distribution (if $p_{i}=1$ for some $i \in \mathbb{N}$ )

2) or a singularly continuous distribution (otherwise).

Proof. 1) Statement 1) follows directly from the fact that the random variables $\left\{\eta_{k}\right\}$ are identically distributed and from a general criterion for the discreteness of the distribution of the random variable $\eta$ (see [11); namely, the distribution of the random variable $\eta$ is purely discrete if and only if $\prod_{k=1}^{\infty} \max _{i} p_{i k}>0$.

2) We prove that if the distribution of the random variable $\eta$ is continuous, then it does not contain an absolutely continuous component. We choose a positive integer $i_{0}$ such that $p_{i_{0}}>0$ (at least one number $i_{0}$ with this property exists) and consider the set

$$
M_{i_{0}}=\left\{x: x \in[0,1], \nu_{i_{0}}\left(x, \bar{O}^{2}\right)=p_{i_{0}}>0\right\} .
$$

By Lemma 4.2, the set $M_{i_{0}}$ is of full $\mu_{\eta}$-measure.

We also consider the set $L_{i_{0}}^{*}=\left\{x: x \in[0,1], \nu_{i_{0}}\left(x, \bar{O}^{2}\right)=0\right\}$. Formula (5) implies that $\lambda\left(L_{i_{0}}^{*}\right)=1$. The sets $M_{i_{0}}$ and $L_{i_{0}}^{*}$ are disjoint. The first of them is the support of the probability measure $\mu_{\eta}$, while the second is the support of Lebesgue measure on the unit interval. Thus the measure $\mu_{\eta}$ is singular with respect to Lebesgue measure, and this is what was to be proved. 
Corollary 4.1. The distribution of the first Ostrogradskiu series with independent identically distributed increments is pure. Moreover, this distribution cannot be absolutely continuous.

Corollary 4.2. All probability measures corresponding to the second Ostrogradskin series with independent identically distributed increments are invariant and ergodic with respect to the one-sided shift transformation $T$ in the $\bar{O}^{2}$-representation. However, none of these measures is absolutely continuous with respect to Lebesgue measure.

As is known, the studies of metric (ergodic) theory of representations of real numbers will significantly simplify if one can find a measure that is invariant and ergodic with respect to the shift transformation in that representation and, at the same time, is absolutely continuous with respect to Lebesgue measure (see [7]). For example, since the Gauss measure (that is, an absolutely continuous probability measure with density $f(x)=\frac{1}{\ln 2} \frac{1}{1+x}$ on the unit interval) is invariant and ergodic with respect to the shift transformation in the representation by continued fractions, one can derive the main ergodic properties of continued fractions (see, for example, [14, 7]).

In conclusion, we show that Corollary 4.2 holds true even if the measure does not necessarily belong to the class of probability measures corresponding to the Ostrogradskil series with independent identically distributed $\bar{O}^{2}$-symbols.

Theorem 4.2. There is no probability measure that is invariant and ergodic with respect to the shift transformation $T$ in the $\bar{O}^{2}$-representation and, at the same time, is absolutely continuous with respect to Lebesgue measure.

Proof. We prove this by contradiction. Assume that there exists an absolutely continuous probability measure $\nu$ that is invariant and ergodic with respect to $T$. Then

$$
\lim _{n \rightarrow \infty} \frac{1}{n} \sum_{j=0}^{n-1} \varphi\left(T^{j}(x)\right)=\int_{0}^{1} \varphi(x) d(\nu(x))=\int_{0}^{1} \varphi(x) f_{\nu}(x) d x
$$

for $\nu$-almost all $x \in[0,1]$ (thus for all sets of positive Lebesgue measure) and for an arbitrary function $\varphi \in L^{1}([0,1], d \nu)$, where $f_{\nu}(x)$ is the density of the measure $\nu$.

Let $\varphi_{i}(x)=1$ if $x \in \Delta_{i}^{\bar{O}^{2}}$ and $\varphi_{i}(x)=0$ otherwise. Then

$$
\int_{0}^{1} \varphi_{i}(x) f_{\nu}(x) d x=\int_{x \in \Delta_{i}^{O^{2}}} f_{\nu}(x) d x>0
$$

for at least one $i \in \mathbb{N}$, say, $i_{0}$.

On the other hand, (5) implies that

$$
\lim _{n \rightarrow \infty} \frac{1}{n} \sum_{j=0}^{n-1} \varphi_{i_{0}}\left(T^{j}(x)\right)=\lim _{n \rightarrow \infty} \frac{N_{i_{0}}(x, n)}{n}=0
$$

for $\lambda$-almost all $x \in[0,1]$. Therefore,

$$
\lim _{n \rightarrow \infty} \frac{N_{i_{0}}(x, n)}{n}=0
$$

for $\lambda$-almost all $x \in[0,1]$ and, at the same time,

$$
\lim _{n \rightarrow \infty} \frac{N_{i_{0}}(x, n)}{n}>0
$$

for a set of positive Lebesgue measure. This contradiction completes the proof of the theorem. 


\section{BIBLIOGRAPHY}

1. S. Albeverio, O. Baranovskyi, M. Pratsiovytyi, and G. Torbin, The Ostrogradsky series and related Cantor-like sets, Acta Arith. 130 (2007), no. 3, 215-230. MR2365703 (2008j:11094)

2. S. Albeverio, O. Baranovskyi, M. Pratsiovytyi, and G. Torbin, The set of incomplete sums of the first Ostrogradsky series and probability distributions on it, Rev. Roum. Math. Pures Appl. 54 (2009), no. 1, 129-145. MR2519524 (2011a:60054)

3. S. Albeverio, I. Pratsiovyta, M. Pratsiovytyi, and G. Torbin, On Bernoulli Convolutions Generated by Second Ostrogradsky Series and their Fine Fractal Properties, SFB-611 Preprint, vol. 459, Bonn University, 2009.

4. K. J. Falconer, Fractal Geometry: Mathematical Foundations and Applications, Wiley, Chichester, 1990. MR.1102677 (92j:28008)

5. O. Jenkinson and M. Policott, Computing the dimension of dynamically defined sets: $E_{2}$ and bounded continued fractions, Ergodic Theory Dynam. Syst. 21 (2001), 1429-1445. MR.1855840 (2003m:37027)

6. T. A. Pierce, On an algorithm and its use in approximating roots of algebraic equations, Amer. Math. Monthly 36 (1929), no. 10, 523-525. MR1521866

7. F. Schweiger, Ergodic Theory of Fibred Systems and Metric Number Theory, Clarendon Press, Oxford, 1995. MR 1419320 (97h:11083)

8. J. O. Shallit, Metric theory of Pierce expansions, Fibonacci Quart. 24 (1986), no. 1, 22-40. MR 825872 (87g:11093)

9. K. G. Valeev and E. D. Zlebov, The metric theory of the Ostrogradskiu algorithm, Ukrain. Mat. Zh. 27 (1975), no. 1, 64-69; English transl. in Ukrainian Math. J. 27 (1975), no. 1, 47-51. MR 0366855 (51:3101)

10. I. M. Prats'ovita, Ostrogradskiu series of second kind and distributions of their incomplete sums, Proceedings of the National Dragomanov Pedagogical University, Physics and Mathematics 7 (2006), 174-189. (Ukrainian)

11. M. V. Prats'ovitiǔ, Fractal Approach to the Studies of Singular Distributions, National Dragomanov Pedagogical University, Kyiv, 1998. (Ukrainian)

12. M. V. Prats'ovitiŭ and O. M. Baranovs'kiı̌, Properties of distributions of random variables with independent differences of consecutive elements of the Ostrogradskiน series, Teor. Imovir. Mat. Stat. 70 (2004), 131-144; English transl. in Theory Probab. Math. Statist. 70 (2005), 147-160. MR2110871 (2005j:11057)

13. E. Ya. Remez, On series with alternating sign which are possibly connected with two algorithms of M. V. Ostrogradski for the approximation of irrational numbers, Uspehi Matem. Nauk (N.S.) 6 (1951), no. 5 (45), 33-42. (Russian) MR0044585 (13:444d)

14. A. Ya. Khinchin, Continued Fractions, Nauka, Moscow, 1978; English transl. of the third (1961) Russian edition, The University of Chicago Press, Chicago-London, 1964. MR514845 (80d:10015), MR0161833(28:5037)

Department of Higher Mathematics, Institute for Physics and Mathematics, National Dragomanov Pedagogical University, Pirogova Street 9, Kyiv 01030, Ukraine

E-mail address: torbin@imath.kiev.ua

Department of Higher Mathematics, Institute for Physics and Mathematics, National Dragomanov Pedagogical University, Pirogova Street 9, Kyiv 01030, Ukraine

E-mail address: lightsoul2008@gmail.com

Received 2/NOV/2009

Translated by N. SEMENOV 